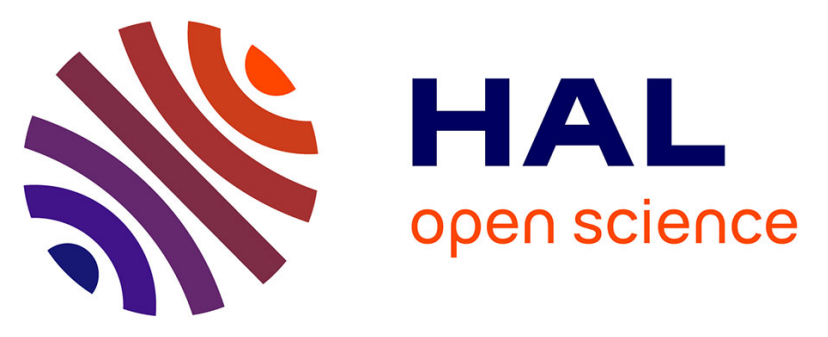

\title{
Optimized bi-resonant nanostructured diode for infrared photodetection through non degenerate two-photon absorption
}

Maxence Dauphin, Baptiste Fix, Grégoire Beaudoin, Isabelle Sagnes, Julien Jaeck, Riad Haïdar

\section{To cite this version:}

Maxence Dauphin, Baptiste Fix, Grégoire Beaudoin, Isabelle Sagnes, Julien Jaeck, et al.. Optimized bi-resonant nanostructured diode for infrared photodetection through non degenerate two-photon absorption. Quantum Sensing and Nano Electronics and Photonics XVII, Feb 2020, San Francisco, United States. pp.Proc. SPIE 11288, 112881S, 10.1117/12.2545730 . hal-02864687

\section{HAL Id: hal-02864687 https://hal.science/hal-02864687}

Submitted on 11 Jun 2020

HAL is a multi-disciplinary open access archive for the deposit and dissemination of scientific research documents, whether they are published or not. The documents may come from teaching and research institutions in France or abroad, or from public or private research centers.
L'archive ouverte pluridisciplinaire HAL, est destinée au dépôt et à la diffusion de documents scientifiques de niveau recherche, publiés ou non, émanant des établissements d'enseignement et de recherche français ou étrangers, des laboratoires publics ou privés. 


\title{
Optimized bi-resonant nanostructured diode for infrared photodetection through non degenerate two-photon absorption
}

\author{
Maxence Dauphin ${ }^{\mathrm{a}}$, Baptiste Fix ${ }^{\mathrm{a}}$, Grégoire Beaudoin ${ }^{\mathrm{b}}$, Isabelle Sagnes ${ }^{\mathrm{b}}$, Julien Jaeck ${ }^{\mathrm{a}}$, and \\ Riad Haïdar ${ }^{\mathrm{a}, \mathrm{c}}$ \\ ${ }^{a}$ ONERA - The French Aerospace Lab, Chemin de la Hunière, 91761 Palaiseau Cedex, France \\ ${ }^{\mathrm{b}}$ Center for Nanoscience and Nanotechnology (C2N) - CNRS, Univ. Paris-Sud, Université \\ Paris-Saclay, 91460 Marcoussis, France \\ cDépartement de Physique, École Polytechnique, Université Paris-Saclay, 91128 Palaiseau, \\ France
}

Two-photon absorption (TPA) is a third order non-linear process that relies on the quasi-simultaneous absorption of two photons. Therefore, it has been proved to be an interesting tool to measure ultra-fast correlations ${ }^{1}$ or to design all-optical switches. ${ }^{2}$ Yet, due to the intrinsically low efficiency of the non-linear processes, these applications rest upon high peak power light sources such as femtosecond and picosecond pulsed laser. However TPA has also been noticed as an appealing new scheme for quantum infrared detection. ${ }^{3,4}$ Indeed, typical quantum detection of IR radiation is based on small gap semiconductors that need to be cooled down to cryogenic temperature to achieve sufficient detectivity. TPA enables the absorption of IR photons by wide gap semiconductors when pump photons are provided to complete optical transitions across the gap. Still, the low efficiency of TPA represents a difficulty to detect usual infrared photon fluxes. To tackle this issue, we combined three strategies to improve the detection efficiency. First, it has been proved theoretically and experimentally that using different pump and signal photon energies, which is known as non degenerate TPA (NDTPA), help increasing the TPA efficiency by several orders of magnitude. ${ }^{5}$ Secondly, it is well known that TPA has a quadratic dependence with the signal electric fields modulus, so we designed a specific nanostructure to enhance the signal field inside the active medium of the detector. Finally, since TPA is a local quasi-instantaneous process, both pump and signal photons must be temporarily and spatially co-localized inside the active medium. We made sure to maximize the overlap of the fields inside our device. In this proceeding, we report the concepts of nanostructures and how it influences TPA absorption in a PIN photodiode. Experimental data point out that infrared photons were detected inside our first generation of diodes. However some issues are still to deal with to reach infrared detection with low fluxes thermal sources. The SNR (signal to noise ratio) can be widely improved by reaching higher values of NDTPA photocurrent and limiting the sub-gap absorptions mainly responsible for the structure noise. Consequently a second generation of nanostructured photodiodes has been designed to perform better detection.

\section{NDTPA theory}

The expected photocurrent generation in a PIN junction through two-photon absorption is a sum of three terms: (1) the linear absorption current, (2) the degenerate TPA current of the pump and (3) the NDTPAgenerated photocurrent :

$$
J_{\text {total }}=\alpha\left(\omega_{p}\right)\left\langle I_{p}\right\rangle+\beta\left(\omega_{p}, \omega_{p}\right)\left\langle I_{p}^{2}\right\rangle+\beta\left(\omega_{p}, \omega_{s}\right)\left\langle I_{p} I_{s}\right\rangle
$$

Where $I_{p}$ (resp. $I_{s}$ ) is the intensity $\left[\mathrm{W} / \mathrm{m}^{2}\right]$ of the pump (resp. signal) beam, $\langle\cdot\rangle$ represent the integration in the i-layer, the $\alpha$ is the linear absorption coefficient and $\beta$ is the TPA coefficient. The first term comes from a linear absorption of sub-bandgap photons from the pump light. This phenomenon has been studied in several materials (silicium, gallium arsenide) and is explained as a photo-assisted Shockley-Read-Hall (PASRH) process. ${ }^{6,7}$ The second term is the degenerate two-photon absorption of the pump. ${ }^{3}$ The last one comes from the non-degenerate

E-Mail : baptiste.fix@onera.fr 
two-photon absorption (NDTPA), which is the absorption of pump and signal photon. The main ambition of this work is to enhance this NDTPA term in order to obtain higher responsivity and SNR. To do so, the top electrode is functionalized with a nanostructure.

\section{Gain through nanostructures}

The NDTPA theory shows that the non-linear absorption is locally proportional to the product of the pump and signal field intensities. So, in every position of the intrinsic zone of the diode, the local charge generation $j_{\mathrm{NDTPA}}$ is driven by the equation $j_{\mathrm{NDTPA}}=\beta I_{p} I_{s}$ where $I_{p}\left[\mathrm{~W} / \mathrm{m}^{2}\right]$ (resp. $I_{s}$ ) is the local pump (resp. signal) intensity. ${ }^{3}$ The nanostructure usefulness is thus to locally concentrate and co-localize the signal and pump fields (see figure 1) inside the intrinsic zone of the PIN junction of the diode where absorption occurs. Taking into account the macroscopic charge collection, the integrated NDTPA photocurrent generation can be written as $J_{\text {NDTPA }}$ as $J_{\text {NDTPA }}=\beta G_{\text {structure }} I_{p}^{(i)} I_{s}^{(i)}$ where $I_{p}^{(i)}$ (resp. $I_{s}^{(i)}$ ) are the incident pump (resp. signal) intensity, and $G_{\text {structure }}$ is the gain due to the nanostructuration defined as :

$$
G_{\text {structure }}=\frac{\left\langle I_{p} I_{s}\right\rangle}{I_{p}^{(i)} I_{s}^{(i)}}
$$

where $\langle\cdot\rangle$ represent the spatial integration in the i-layer over a period of the nanostructure. This theoretical gain is easily computed using the linear B-Spline modal method ${ }^{3,8}$ and is used as a figure of merit for the design optimization.
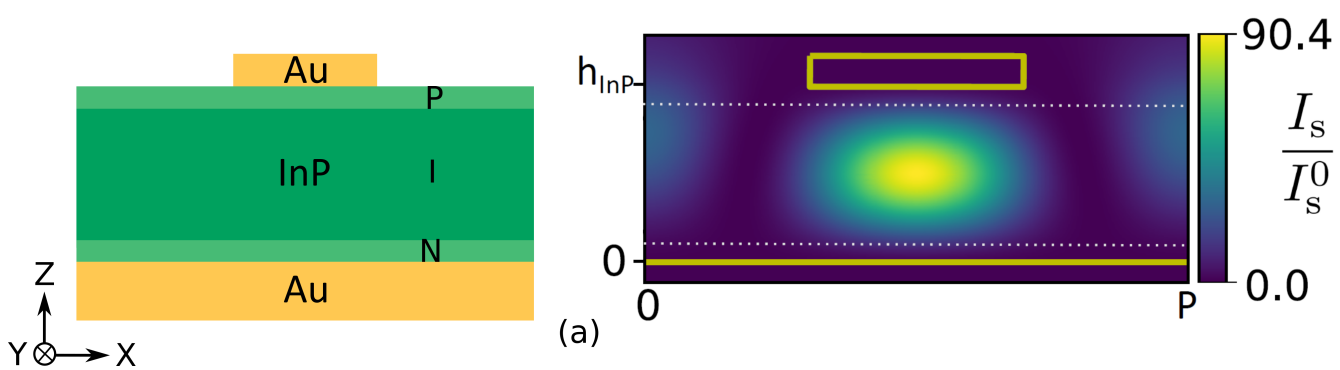

(b)

Figure 1. (a) Cross section of a nanostructured photodiode. (b) Signal field intensity for TE polarisation at $3.39 \mu \mathrm{m}$. The nanostructure is able to focus the electric field inside the intrinsic zone of the PIN photodiode.

\section{Experimental setup}

The experimental setup, represented on figure 2, consists of three elements : the signal source, the pump source and the reflectivity measurement arm. The signal source is a M-Squared Firefly-IR optical parametric oscillator, delivering pulses of duration $t=10 \mathrm{~ns}$ under a repetition rate of $\mathrm{f}=150 \mathrm{kHz}$. It is coupled to a fiber and its linear polarization is controlled through a combination of a polarizer and a half-wave plate. An aspheric lens (focal length $6 \mathrm{~mm}$ ) is used to focus the light into a spot of diameter $25 \mu \mathrm{m}$ (measured at $\frac{1}{e^{2}}$ by a knife-edge method) on the photodiode. The pump light is emitted by a fibered continuous wave laser diode (Thorlabs LPS1060-FC) that is also linearly polarized. The pump beam is decentered from the focusing lens in order to modify the beam angle of incidence. The whole optical cage is mounted on a motorized three axis translation stage on top of a CascadeMicrotech EP9 probe station. The diode is connected by metallic probe to a sub-femtoamp sourcemeter Keithley 6430 which applies a bias voltage of $-100 \mathrm{mV}$ and collects the generated photocurrent. All the photocurrent measurements have been achieved at room temperature.

\section{Infrared detection in a $1^{\text {st }}$ generation of nanostructured photodiode}

A first structure has been designed for two-photon absorption with a gain numerically calculated using the linear B-Spline modal method. ${ }^{3,8}$ The periodic $1 \mathrm{D}$ structure parameters, detailed in figure 3, are $h_{\mathrm{Au}}=100 \mathrm{~nm}$, 


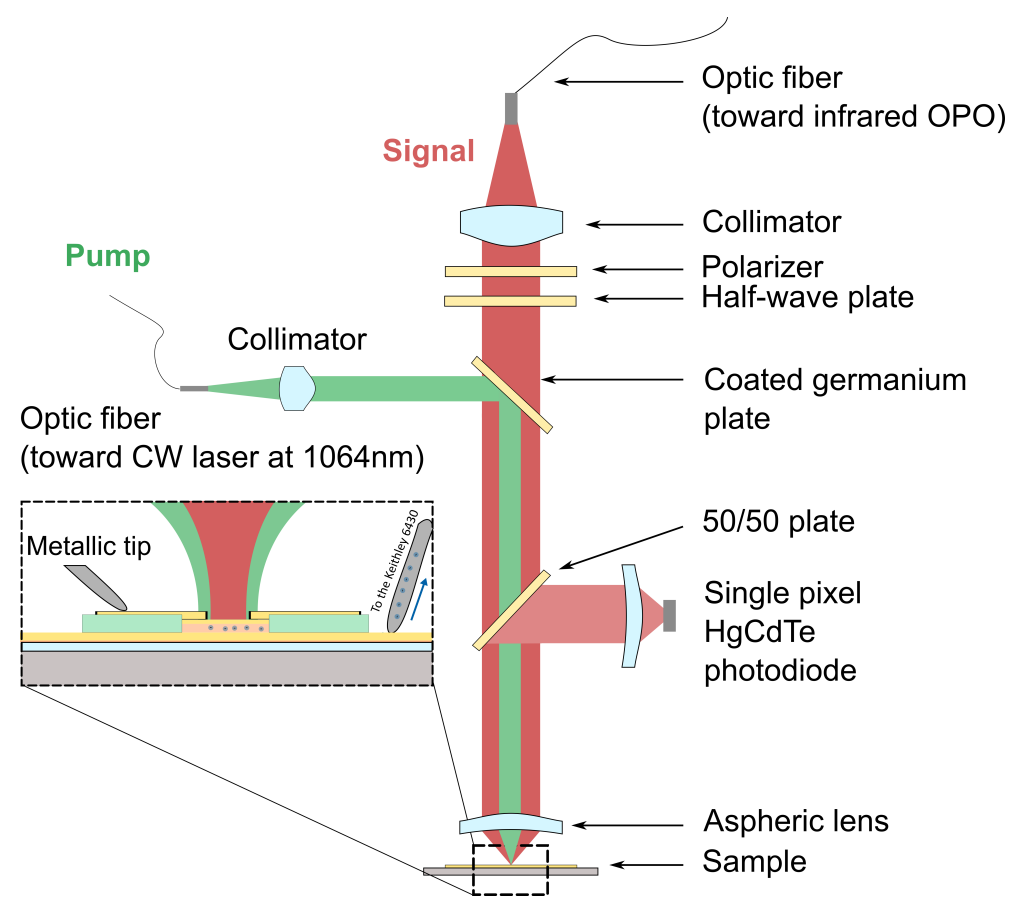

Figure 2. Schematic of the dedicated bench. The sample is fixed to the table and the whole optical cage can be translated along the three axis X,Y,Z.

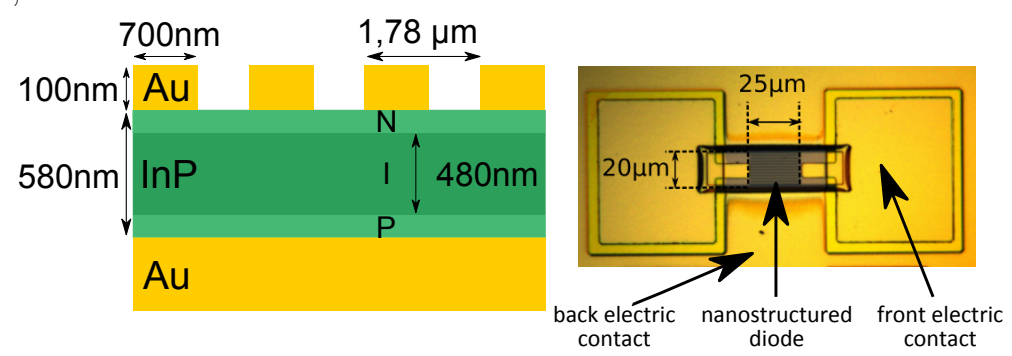

Figure 3. On the left is a cross sectional schematic of the diode. An indium phosphide PIN diode of $480 \mathrm{~nm}$ non intentionally doped zone is placed between a back mirror and a front nanostructured electrode. On the right is an annotated picture taken with a microscope from one realization of the diode. In the center is the nanostructured electrode of dimension $20 \times 25 \mu \mathrm{m}$. On both sides are gold pads to place the metallic tip and achieve one of the electric contacts.

$\mathrm{L}=700 \mathrm{~nm}, \mathrm{p}=1780 \mathrm{~nm}$, and $\mathrm{w}=580 \mathrm{~nm}$. The nominal thicknesses of the $\mathrm{p}$-i-n junction are $50 \mathrm{~nm}$ for the p-layer, $480 \mathrm{~nm}$ for the i-layer, and $50 \mathrm{~nm}$ for the n-layer. The $\mathrm{n}(\mathrm{p})$-layer are dopped with a density of $1.10^{18} \mathrm{~cm}^{-3}$ while the intrinsic zone has an n-type residual doping with a density about $1.10^{16} \mathrm{~cm}^{-3}$. For TE polarized wave, this structure exhibits a guided mode. ${ }^{9,10}$ The best signal resonance within this device is reached at $\lambda=3.39 \mu \mathrm{m}$ due to the existence of a delocalized mode inside the diode. Regarding the pump resonance the field repartition inside the intrinsic zone depends on the incidence angle. The best coupling between the pump light at $1.06 \mu \mathrm{m}$ and the device is obtained under $11^{\circ}$ incidence. The measured photocurrent generated by NDTPA is about 40 pA with an experimental gain of $G=24$ from nanostructures, reaching a SNR of 20 (see figure 4).

As a result this first generation of nanostructured photodiodes managed to validate the key concepts of infrared detection for NDTPA. However some issues are still to be tackled with to reach infrared detection with low fluxes thermal sources. The SNR (signal to noise ratio) can be widely improved by reaching higher values of NDTPA photocurrent and limiting the structure noise due to sub-gap absorptions. So the next step is to improve the detection inside the new incoming structures.

How to design the $2^{\text {nd }}$ generation of nanostructured photodiodes ? 

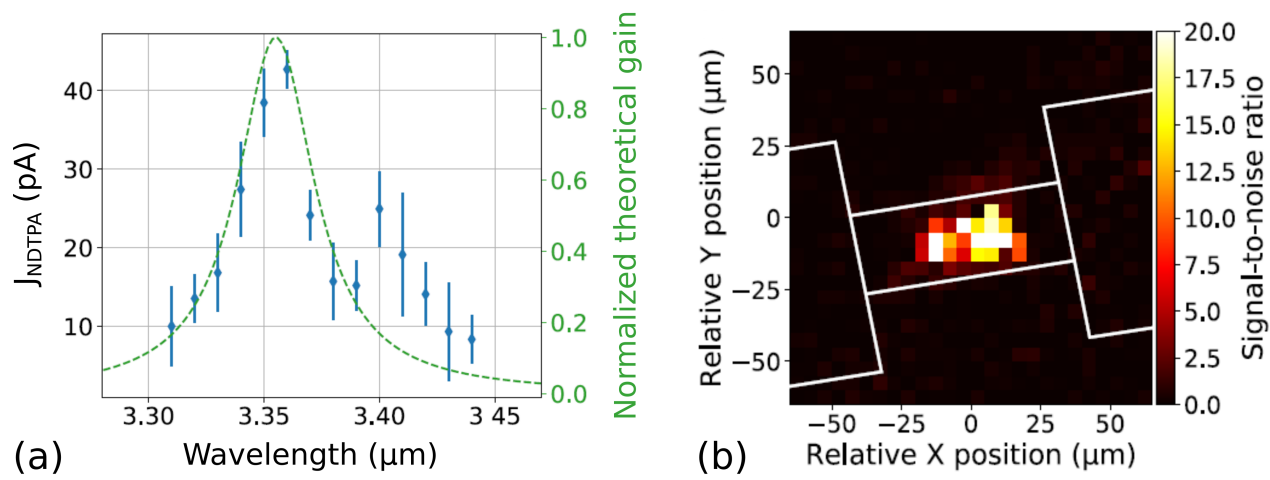

Figure 4. (a) Spectral evolution of the NDTPA-generated photocurrent for mean incident power of $300 \mu \mathrm{W}$ for the pump and $1.4 \mathrm{~mW}$ for the signal. The measurements follow the theoretical linear gain of the nanostructure. We attribute the re-bounce to the signal beam angular spectrum. (b) Scanned map of the signal-to-noise ratio measured for a TE polarized signal at $3.36 \mu \mathrm{m}$. The incident signal mean power is $1.4 \mathrm{~mW}$ and the incident pump mean power is $1.3 \mathrm{~mW}$. The white line is the diode skeleton as measured simultaneously through reflection that has been added as a guide for the eyes.

Based on the first generation insights, two paths have been prioritized to improve the photodiodes design. The first one is to enhance the ND2PA generation through a higher gain G. Thus the structure should be resonant for both the pump and the signal beams to improve the co-localization and the gain factor. The second path is to decrease the noise in the structures through the reduction of the PASRH effect which can be achieved by the use of new figures of merits to strengthen detection.

\section{Improving the SNR}

The photocurrent generated by PASRH is 10 times higher than the NDTPA one in the first generation and is by far the main noise source of our system. PASRH is a linear phenomenon when the pump photon is absorbed in a sub-gap energy (induced by defects in the crystal) and the optical transition between valence and conduction bands is completed due to thermal energy. While PASRH is the main noise source and needs to be curtailed, the pump photons are still needed to trigger NDTPA. Consequently the challenge for two-photon detection is to maximize the co-localization between signal and pump fields within the structure in order to favor NDTPA while decreasing the zones with pump field but without signal (which leads to noise generation). So in order to minimize the PASRH and improve NDTPA, a new figure of merit has been designed :

$$
F=\frac{\left\langle\widetilde{I}_{p} I_{s}\right\rangle}{\left\langle\widetilde{I}_{p}^{2}\right\rangle \cdot\left\langle\widetilde{I}_{p} \bar{S}_{s}\right\rangle}
$$

Where $\langle\cdot\rangle$ represents the integration in the i-layer, $\widetilde{I}_{p}$ is the normalized pump field, and $\bar{S}_{s}$ is a binary function equal to 1 when the signal field amplitude is below the threshold value ( $20 \%$ of the max). $\mathrm{F}$ combine three terms : $\left\langle\widetilde{I}_{p} I_{s}\right\rangle$ is the NDTPA, $\left\langle\widetilde{I}_{p}^{2}\right\rangle$ restrains the pump concentration so DTPA is limited and $\left\langle\widetilde{I}_{p} \bar{S}_{s}\right\rangle$ favor the structures that tend to disfavor PASRH by minimizing the zone with only pump field and without signal field.

\section{Minimizing angle influence}

Another experimental problem was the sensibility of the structure with the beam angle of incidence. Indeed, both beam are focalized by the experimental bench photodetection which is, at the end of the day, the closest experimental imitation of an infrared camera. It thus appears important for the nanostructure to have as high an angular acceptance as possible. The figure of merit has been adapted to take this consideration into account :

$$
F=\frac{\left\langle\widetilde{\widehat{I}}_{p} I_{s}\right\rangle}{\left\langle\widetilde{\widehat{I}}_{p}^{2}\right\rangle \cdot\left\langle\widetilde{\widehat{I}}_{p} \bar{S}_{s}\right\rangle} \text { with } \widehat{I}=\left|\sum_{\theta_{\min }}^{\theta_{\max }} a_{\theta} E_{p}(\theta)\right|^{2}
$$




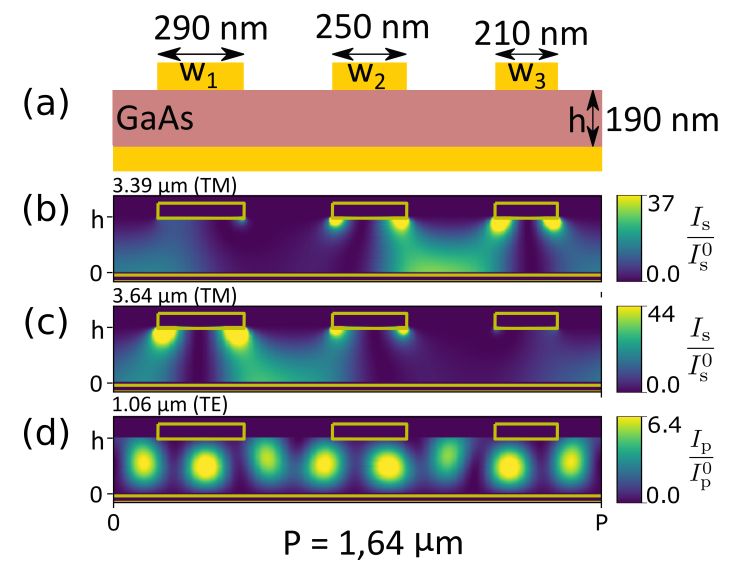

(e)

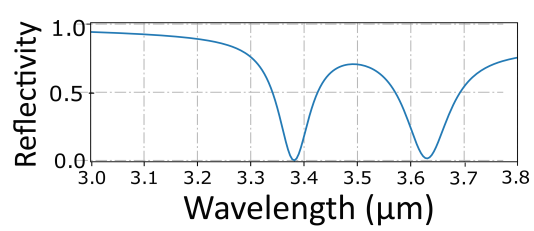

(f)

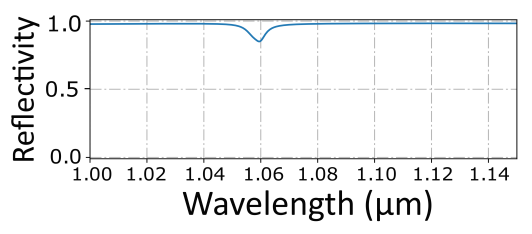

Figure 5. Results of the optimized bi-resonant structure nFPc-GMR. (a) Cross section of a structure period. Field intensity along the structure (b) at $3.39 \mu \mathrm{m}$ and (c) at $3.64 \mu \mathrm{m}$ for TM polarization. (d) Pump field intensity for TE polarization. (e) Reflectivity spectrum of the structure for TM polarized signal. (f) and for TE polarized pump.

\begin{tabular}{|c|c|c|}
\hline & $1^{\text {st }}$ generation & $2^{\text {nd }}$ generation \\
\hline $\mathrm{F}$ & 35 & 196 \\
\hline$F_{\text {foc }}$ & 0.83 & 172 \\
\hline $\mathrm{G}$ & 40 & 1810 \\
\hline
\end{tabular}

Table 1. Figure of merits values of different generations. The second generation is more optimized for two-photon absorption than the first one since the values are higher.

\section{Design of the next generation of nanostructure}

The second generation of nanostructured photodiodes has been optimized for NDTPA with a wavelength pump of $1.06 \mu \mathrm{m}$ and a wavelength signal between 3 and $4 \mu \mathrm{m}$. A new type of nano-cavity is used to allow a thick semiconductor PIN junction and is optimized to achieved the highest figures of merits. It is called a coupled nano Fabry-Perot $(\mathrm{nFPc})$ resonance. It consists of a three waves destructive interference and is enabled thanks to a coupling of two nano Fabry-Perot. GaAs and InP are both used because of their favorable twophoton absorption coefficient $\beta$. The $\mathrm{nFPc}$ structure has been designed and optimized with BMM. The periodic 1D structure parameters are detailed in figure 5. As a matter of comparison, the figures of merits of each photodiodes generation are displayed in Table 1. The new design achieve better values compared to the first generation due to many reasons. At first the new junction has been designed to have the higher possible gain so two-photon absorption is even more favored. This comes from the bi-resonant structure that leads to a better co-localization of pump and signal. Then the design is all about increasing SNR to ensure NDTPA detection thanks to figure of merits. Finally, the nFPc resonance used is a new high-quality factor resonator that opens new paths for further interesting structures.

The structures have been fabricated in the clean room facilities of the C2N. Each sample contains $8 \times 14$ photodiodes with a $50 \mu \mathrm{m} \times 60 \mu \mathrm{m}$ absorbing area. The preliminary electrical and optical characterisation have been achieved (see figure 6). The electrical characterization of the PIN junctions are done with a Keithley 6430. The optical characterization has been achieved using a Brüker Hyperion-microscope.

In summary, a nanostructured infrared photodetector through non-degenerated two-photon absorption has been designed, fabricated and characterized. The detection of a $3.39 \mu \mathrm{m}$ nanosecond pulsed signal is demonstrated at room temperature inside a thin indium phosphide PIN junction with a signal-to-noise ratio of 15 thanks to a gain of 24 due to the nanostructure. A detailed study of the limitations to tackle, leads to the design of a second generation which favors NDTPA over other competitive sub-bandgap absorption and which reaches a theoretical gain of 1810. ${ }^{11}$ By enabling a clear increase of the NDTPA responsivity, the nanostructuration of wide-gap semiconductor based photodiodes paves the way toward continuous wave infrared detection at room temperature. 

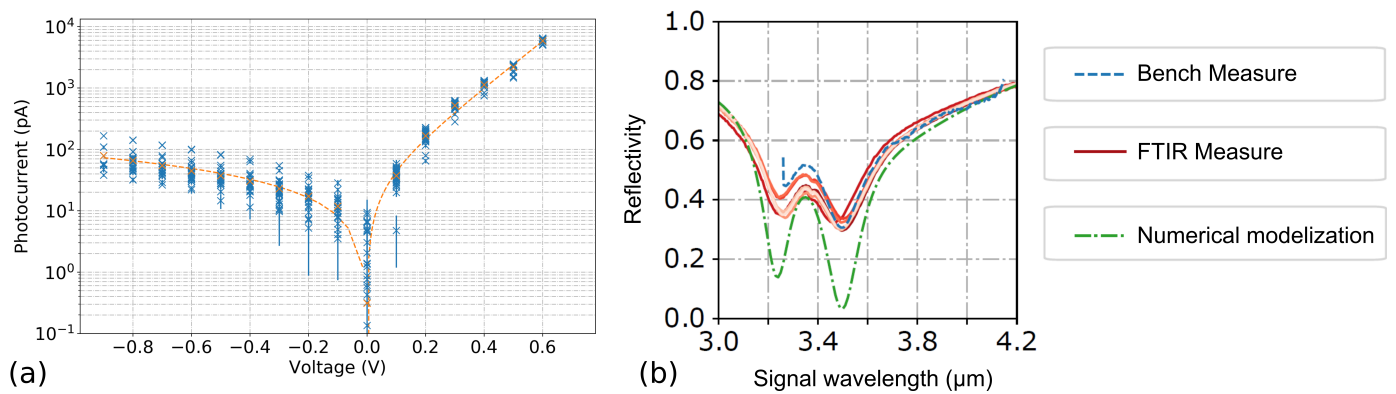

Figure 6. (a) Electrical characterization of a GaAs photodiode sample. The structure behaves like a diode so the structure is electrically functional. 12 photodiodes I-V curves are displayed (b) Reflectivity curve of the GaAs photodiodes. Numerical simulations are continuous curves and experimental ones are dashed.

Acknowledgments The research was supported by a DGA-MRIS scholarship and was partially supported by the French RENATECH network. The authors thanks Jean-luc Pelouard for his support and Christophe Dupuis and Nathalie Bardou for their technological support in the fabrication processes.

\section{REFERENCES}

[1] F. Boitier, A. Godard, E. Rosencher, and C. Fabre, "Measuring photon bunching at ultrashort timescale by two-photon absorption in semiconductors," Nature Physics 5(4), pp. 267-270, 2009.

[2] S. M. Hendrickson, C. N. Weiler, R. M. Camacho, P. T. Rakich, A. I. Young, M. J. Shaw, T. B. Pittman, J. D. Franson, and B. C. Jacobs, "All-optical-switching demonstration using two-photon absorption and the zeno effect," Phys. Rev. A 87, p. 023808, Feb 2013.

[3] B. Portier, B. Vest, F. Pardo, N. Péré-Laperne, E. Steveler, J. Jaeck, C. Dupuis, N. Bardou, A. Lemaître, E. Rosencher, R. Haïdar, and J.-L. Pelouard, "Resonant metallic nanostructure for enhanced two-photon absorption in a thin gaas p-i-n diode," Applied Physics Letters 105(1), p. 011108, 2014.

[4] D. Fishman, C. M. Cirloganu, S. Websterand, L. A. Padilha, M. Monroe, D. J. Hagan, and E. W. V. Stryland, "Sensitive mid-infrared detection in wide-bandgap semiconductors using extreme non-degenerate two-photon absorption," Nature Photonics 5, pp. 561-565, 2011.

[5] C. M. Cirloganu, L. A. Padilha, D. A. Fishman, S. Webster, D. J. Hagan, and E. W. V. Stryland, "Extremely nondegenerate two-photon absorption in direct-gap semiconductors," Opt. Express 19, pp. 22951-22960, Nov 2011.

[6] B. Vest, E. Lucas, J. Jaeck, R. Haïdar, and E. Rosencher, "Silicon sub-bandgap photon linear detection in two-photon experiments: A photo-assisted shockley-read-hall mechanism," Applied Physics Letters 102(3), p. 031105, 2013.

[7] B. Vest, B. Fix, J. Jaeck, and R. Haïdar, "Competition between sub-bandgap linear detection and degenerate two-photon absorption in gallium arsenide photodiodes," Journal of the European Optical Society-Rapid Publications 12(1), p. 26, 2016.

[8] P. Bouchon, F. Pardo, R. Haïdar, and J.-L. Pelouard, "Fast modal method for subwavelength gratings based on b-spline formulation," J. Opt. Soc. Am. A 27, pp. 696-702, Apr 2010.

[9] R. Magnusson and S. S. Wang, "New principle for optical filters," Applied Physics Letters 61(9), pp. 10221024, 1992.

[10] E. Sakat, G. Vincent, P. Ghenuche, N. Bardou, S. Collin, F. Pardo, J.-L. Pelouard, and R. Haïdar, "Guided mode resonance in subwavelength metallodielectric free-standing grating for bandpass filtering," Opt. Lett. 36, pp. 3054-3056, Aug 2011.

[11] B. Fix, Diodes nanostructurées pour la détection infrarouge par absorption à deux photons. PhD thesis, Université Paris-Saclay - Ecole Polytechnique, 2018. 